Advances in

Applied Clifford Algebras

\title{
Best Pair of Two Skew Lines over the Octonions
}

\author{
P. Saraiva, P.D. Beites*, J. Fernandes, C. Costa and José Vitória
}

\begin{abstract}
This is a work on an application of octonions to Analytic Geometry. In the octonionic context, the orthogonal projection of a point onto a straight line is presented. Further, the best approximation pair of points of two skew lines over the octonions is studied.
\end{abstract}

Keywords. Octonion equation of a line, orthogonal projection, inner product of octonions, parallel and perpendicular octonions, double vector cross product in $\mathbb{R}^{7}$, best approximation pair.

\section{Introduction}

A concept that is deeply related to the vector cross product in the real linear space $\mathbb{R}^{7}$ is that of a real composition algebra. Concretely, we have a real algebra $(U, *)$ endowed with a nondegenerate quadratic form (the norm) $N$ : $U \rightarrow \mathbb{R}$ which is multiplicative, i.e., for any $x, y \in U$,

$$
N(x * y)=N(x) N(y) .
$$

From the norms of these type of algebras, that can be either anisotropic or isotropic, arises a dichotomy: either they are division algebras or split algebras, respectively. Assuming anisotropy, Hurwitz Theorem, generalized in [6], asserts that the only finite dimensional real composition algebras with identity are, up to isomorphism, the non-split ones of the: real numbers, $\mathbb{R}$; complex numbers, $\mathbb{C}$; quaternions, $\mathbb{H}$; octonions, $\mathbb{D}$. Consequently, as it was shown in [4], if it is possible to define a vector cross product in the real Euclidean space $\mathbb{R}^{n}$, satisfying the same requirements as the usual one considered in $\mathbb{R}^{3}$, then $n=1$ (trivial case), $n=3$ or $n=7$.

Let us recall that the usual real linear space $\mathbb{R}^{7}$ can be regarded as the real linear space $\operatorname{Im} \mathbb{D}$ of the pure octonions and, indeed, one can formulate the definition of the vector cross product $\times$ in $\mathbb{R}^{7}$ in terms of the product of octonions. These hypercomplex numbers play an important role both in

*Corresponding author. 
Mathematics and in Physics. For instance, in [3], connections with Clifford algebras, spinors, Bott periodicity, projective and Lorentzian geometry, Jordan algebras, exceptional Lie groups, quantum logic, special relativity and supersymmetry are described. Some motivations and applications for nonassociative Physics may also be found in [13].

During the last years, a considerably amount of work pointed out the possibility that the nature may have more than the common four time-space dimensions (e.g., Kaluza-Klein theories, [1]). The extra dimensions are unobservable thanks to their compactness, but they are particularly relevant in the framework of the Standard-Model for particle physics and the attempts to solve the so called hierarchy problem (e.g., [2], [14], [12]). The hierarchy problem concerns the fact that it is not known why the weak force in the Universe is much stronger (typically $10^{32}$ times more) than the gravity force. Recently, it was shown in [11] that if the fundamental particles, the fermions, are assumed to have seven time-spatial dimensions then the above problem could be solved.

In this work, within the octonionic context, we consider an Analytic Geometry problem that once was dealt in [16] concerning quaternions. We obtain, in Section 3, the orthogonal projection of the origin of the coordinates onto an octonion equation of a straight line. Moreover, taking the norm of the pure octonion, belonging to the given line, which is closest to the origin, we find the distance from the mentioned point to this line.

The cited problem is then extended, in Section 4, to the case of the orthogonal projection of an external point onto a line over the octonions. One possible approach would be to use an adequate translation, the results in Section 3 and, at the end of the process, the inverse translation. Instead of this, we give a geometric construction where properties of the octonions are applied.

Finally, the best pair of two skew lines over the octonions is obtained in Section 5. In other words, we present the points defining the shortest segment connecting two skew lines over the octonions, allowing us to compute the distance between those lines. As our ground space is an inner product one, by [7], we have guaranteed the existence and uniqueness of the best approximation points we are looking for.

Since we apply properties of the product of octonions and of the cross product of vectors in $\mathbb{R}^{7}$ throughout the work, several results on octonions and some specification for pure octonions are needed. Furthermore, important technical role is played by the inner product of two octonions and by the double cross product of vectors in $\mathbb{R}^{7}$. This set of notions and results is exposed in the next section.

\section{Preliminaries}

In this section, our main sources are the works [8], [9] and [16]. 


\subsection{The product of octonions}

Here are presented two procedures for multiplying octonions: a table and a matrix multiplication.

Let $(\mathbb{D}, *)$ be the classical real algebra of the octonions with basis $\left\{e_{0}, \ldots, e_{7}\right\}$ and multiplication table

\begin{tabular}{|r|r|r|r|r|r|r|r|r|}
\hline$*$ & $e_{0}$ & $e_{1}$ & $e_{2}$ & $e_{3}$ & $e_{4}$ & $e_{5}$ & $e_{6}$ & $e_{7}$ \\
\hline$e_{0}$ & $e_{0}$ & $e_{1}$ & $e_{2}$ & $e_{3}$ & $e_{4}$ & $e_{5}$ & $e_{6}$ & $e_{7}$ \\
\hline$e_{1}$ & $e_{1}$ & $-e_{0}$ & $e_{3}$ & $-e_{2}$ & $e_{5}$ & $-e_{4}$ & $e_{7}$ & $-e_{6}$ \\
\hline$e_{2}$ & $e_{2}$ & $-e_{3}$ & $-e_{0}$ & $e_{1}$ & $e_{6}$ & $-e_{7}$ & $-e_{4}$ & $e_{5}$ \\
\hline$e_{3}$ & $e_{3}$ & $e_{2}$ & $-e_{1}$ & $-e_{0}$ & $-e_{7}$ & $-e_{6}$ & $e_{5}$ & $e_{4}$ \\
\hline$e_{4}$ & $e_{4}$ & $-e_{5}$ & $-e_{6}$ & $e_{7}$ & $-e_{0}$ & $e_{1}$ & $e_{2}$ & $-e_{3}$ \\
\hline$e_{5}$ & $e_{5}$ & $e_{4}$ & $e_{7}$ & $e_{6}$ & $-e_{1}$ & $-e_{0}$ & $-e_{3}$ & $-e_{2}$ \\
\hline$e_{6}$ & $e_{6}$ & $-e_{7}$ & $e_{4}$ & $-e_{5}$ & $-e_{2}$ & $e_{3}$ & $-e_{0}$ & $e_{1}$ \\
\hline$e_{7}$ & $e_{7}$ & $e_{6}$ & $-e_{5}$ & $-e_{4}$ & $e_{3}$ & $e_{2}$ & $-e_{1}$ & $-e_{0}$ \\
\hline
\end{tabular}

TABLE 1. Octonion multiplication table, [8].

where $e_{0}$ is the identity element. From the above table, by bilinearity, the product of any two octonions can be obtained. Recall also that $\mathbb{D} \cong \mathbb{R}^{8}$ and, so, the elements in $\left\{e_{0}, e_{1}, \ldots, e_{7}\right\}$ can be identified with the ones in the canonical basis $\{(1,0,0 \ldots, 0),(0,1,0, \ldots, 0), \ldots,(0,0, \ldots, 0,1)\}$ of the usual real vector space $\mathbb{R}^{8}$.

In the exhibited table, it is possible to observe that: $e_{i}$ and $e_{j}$ anticommute for $i \neq j ; e_{1}, \ldots, e_{7}$ are square roots of $-e_{0}$. Together with two rules (the last observation; $e_{0}$ is the identity), the Fano plane mnemonic totally describes the algebra structure of the octonions. In the figure below we present the Fano diagram for the previous octonion multiplication table, where the cyclic ordering of each three elements lying on the same line is shown by the arrows.

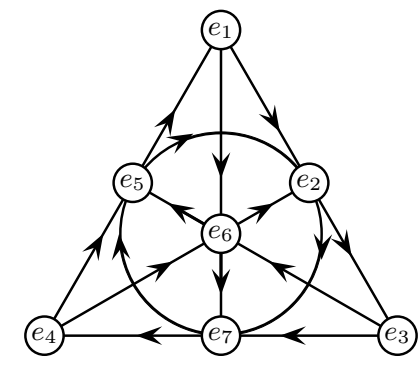

FigURE 1. Fano plane for the octonion multiplication in Table 1 .

See [3] for more details, namely the fact that the Fano plane is the projective plane over the field $\mathbb{Z}_{2}=\{\overline{0}, \overline{1}\}$, which leads to a certain picture of the octonions. Furthermore, the mentioned reference describes other constructions of the octonions: through the Cayley-Dickson process; using Clifford algebras. 
Hereinafter, in order to simplify notations, $e_{0}$ will be denoted by 1 , which in turn will be omitted most times and so a generic octonion $g$ will simply be written as

$$
g=g_{0}+\sum_{i=1}^{7} g_{i} e_{i}, \text { where } g_{j} \in \mathbb{R} \text { for } j \in\{0, \ldots, 7\} .
$$

In order to determine the product $a * b$ of two octonions, instead of using the multiplication table, the ordinary multiplication of two convenient matrices as in [8] may be used:

$$
a * b=\left[\begin{array}{rrrrrrrr}
a_{0} & -a_{1} & -a_{2} & -a_{3} & -a_{4} & -a_{5} & -a_{6} & -a_{7} \\
a_{1} & a_{0} & -a_{3} & a_{2} & -a_{5} & a_{4} & -a_{7} & a_{6} \\
a_{2} & a_{3} & a_{0} & -a_{1} & -a_{6} & a_{7} & a_{4} & -a_{5} \\
a_{3} & -a_{2} & a_{1} & a_{0} & a_{7} & a_{6} & -a_{5} & -a_{4} \\
a_{4} & a_{5} & a_{6} & -a_{7} & a_{0} & -a_{1} & -a_{2} & a_{3} \\
a_{5} & -a_{4} & -a_{7} & -a_{6} & a_{1} & a_{0} & a_{3} & a_{2} \\
a_{6} & a_{7} & -a_{4} & a_{5} & a_{2} & -a_{3} & a_{0} & -a_{1} \\
a_{7} & -a_{6} & a_{5} & a_{4} & -a_{3} & -a_{2} & a_{1} & a_{0}
\end{array}\right]\left[\begin{array}{l}
b_{0} \\
b_{1} \\
b_{2} \\
b_{3} \\
b_{4} \\
b_{5} \\
b_{6} \\
b_{7}
\end{array}\right] .
$$

\subsection{Conjugate, norm and inverse of octonions}

Here some notations and results on octonions are collected. We write an octonion $p$ as the sum of its scalar part $S(p)$ and its vector part $\vec{V}(p)$ :

$$
p=p_{0}+\sum_{i=1}^{7} p_{i} e_{i}=p_{0}+\vec{p}=S(p)+\vec{V}(p) .
$$

A few particularizations for pure octonions, that is, octonions with scalar part equal to 0 , are listed.

The conjugate of an octonion $p$ is $p^{c}=p_{0}-\vec{p}=S(p)-\vec{V}(p)$, the norm of $p$ is $N(p)=p * p^{c}=p^{c} * p$ and the inverse of $p(\neq 0)$ is $p^{-1}=\frac{1}{N(p)} p^{c}$.

We have also, for any $p, q \in \mathbb{D}$, the following properties:

$$
(p+q)^{c}=p^{c}+q^{c},(p * q)^{c}=q^{c} * p^{c}, N(p * q)=N(p) N(q) .
$$

Next we specify some notions and properties of octonions to the case of pure octonions. For every pure octonion $p=0+\vec{p}=0+\vec{V}(p)$, we have:

$$
(\vec{p})^{c}=-\vec{p},(\vec{p} * \vec{q})^{c}=\vec{q} * \vec{p} \text { and } N(\vec{p})=-\vec{p} * \vec{p} .
$$

If in addition we have $\vec{p} \neq \overrightarrow{0}$, then:

$$
(\vec{p})^{-1}=-\frac{1}{N(\vec{p})} \vec{p},\left[(\vec{p})^{-1}\right]^{c}=-(\vec{p})^{-1}, N\left[(\vec{p})^{-1}\right]=\frac{1}{N(\vec{p})},(\vec{p})^{-1} *(\vec{p})^{-1}=-\frac{1}{N(\vec{p})} .
$$

\subsection{Parallelism and orthogonality of octonions}

After defining an inner product of octonions, it is natural to think on the angle between two octonions. Here we restrict ourselves to two extreme cases: parallel and perpendicular octonions. 
Definition 2.1. [16] The inner product $\langle p, q\rangle$ of two octonions $p$ and $q$ is given by

$$
<p, q>=S\left(p * q^{c}\right) .
$$

Further, two octonions $p$ and $q$ are perpendicular (or orthogonal) if $S\left(p * q^{c}\right)=$ 0 and parallel if $\vec{V}\left(p * q^{c}\right)=\overrightarrow{0}$.

We can now state one more property for any two pure distinct octonions $\vec{p}$ and $\vec{q}$ :

$$
\vec{p} * \vec{q}=-\vec{q} * \vec{p} \text { if and only if } \vec{p}, \vec{q} \text { are perpendicular. }
$$

Definition 2.2. [16] Two octonions $p$ and $q$ have parallel vector parts if $\vec{V}(p)=$ $\alpha \vec{V}(q)$ for some $\alpha \in \mathbb{R}$.

Notice that the non-parallel octonions $1+e_{1}$ and $-e_{1}$ have parallel vector parts. So, the definitions of parallel octonions and the preceding one are not equivalent. However, as we will see in Subsection 2.5, they coincide for pure octonions.

\subsection{Alternativity of octonions}

Despite the non-associativity of octonions, a weak form of associativity holds.

Theorem 2.3. [16] The algebra $(\mathbb{D}, *)$ is alternative, that is, for any octonions $p$ and $q$,

$$
(p * p) * q=p *(p * q) \text { and } \quad q *(p * p)=(q * p) * p .
$$

Beyond the well known previous theorem, we recall that the alternativity of an algebra implies the validity of the Moufang identities, [16]. In particular, for any octonions $p, q$ and $r$,

$$
(p * q) *(r * p)=p *(q * r) * p .
$$

Both results will be needed in the search for the best approximation point.

\subsection{Vectors of $\mathbb{R}^{7}$ as pure octonions}

We have to deal, in this work, with vectors in the usual real vector space $\mathbb{R}^{7}$ equipped with the corresponding Euclidean metric $\|\cdot\|$. Since the space $\operatorname{Im} \mathbb{D}=\{q \in \mathbb{D}: S(q)=0\}$ of the pure octonions can be isomorphically identified with $\mathbb{R}^{7}$, we do some operations with the mentioned vectors considering them either as pure octonions or as vectors. So the usual products of vectors are presented and some identities are taken into consideration. For the dot, cross and triple scalar products in $\mathbb{R}^{7}$, we again favour the matrix approach. The usual notations, where $B_{c_{7}}=\left\{\vec{e}_{1}, \ldots, \vec{e}_{7}\right\}$ stands for the canonical basis of $\mathbb{R}^{7}$, are considered.

$$
\vec{m}=\sum_{i=1}^{7} m_{i} \vec{e}_{i}=\left[\begin{array}{c}
m_{1} \\
m_{2} \\
m_{3} \\
\vdots \\
m_{7}
\end{array}\right] \text { with } \vec{e}_{1}=\left[\begin{array}{c}
1 \\
0 \\
0 \\
\vdots \\
0
\end{array}\right], \vec{e}_{2}=\left[\begin{array}{c}
0 \\
1 \\
0 \\
\vdots \\
0
\end{array}\right], \ldots, \vec{e}_{7}=\left[\begin{array}{c}
0 \\
0 \\
0 \\
\vdots \\
1
\end{array}\right]
$$


i) Vector dot product

We have

$$
\vec{p} \bullet \vec{q}=\sum_{i=1}^{7} p_{i} q_{i}
$$

ii) Vector cross product

For the cross product of two vectors in $\mathbb{R}^{7}$, we present a multiplication table for the unit vectors in $B_{c_{7}}$ and also a matrix procedure.

a) The table is the subsequent one.

\begin{tabular}{|r|r|r|r|r|r|r|r|}
\hline$\times$ & $\vec{e}_{1}$ & $\vec{e}_{2}$ & $\vec{e}_{3}$ & $\vec{e}_{4}$ & $\vec{e}_{5}$ & $\vec{e}_{6}$ & $\vec{e}_{7}$ \\
\hline$\vec{e}_{1}$ & $\overrightarrow{0}$ & $\vec{e}_{3}$ & $-\vec{e}_{2}$ & $\vec{e}_{5}$ & $-\vec{e}_{4}$ & $\vec{e}_{7}$ & $-\vec{e}_{6}$ \\
\hline$\vec{e}_{2}$ & $-\vec{e}_{3}$ & $\overrightarrow{0}$ & $\vec{e}_{1}$ & $\vec{e}_{6}$ & $-\vec{e}_{7}$ & $-\vec{e}_{4}$ & $\vec{e}_{5}$ \\
\hline$\vec{e}_{3}$ & $\vec{e}_{2}$ & $-\vec{e}_{1}$ & $\overrightarrow{0}$ & $-\vec{e}_{7}$ & $-\vec{e}_{6}$ & $\vec{e}_{5}$ & $\vec{e}_{4}$ \\
\hline$\vec{e}_{4}$ & $-\vec{e}_{5}$ & $-\vec{e}_{6}$ & $\vec{e}_{7}$ & $\overrightarrow{0}$ & $\vec{e}_{1}$ & $\vec{e}_{2}$ & $-\vec{e}_{3}$ \\
\hline$\vec{e}_{5}$ & $\vec{e}_{4}$ & $\vec{e}_{7}$ & $\vec{e}_{6}$ & $-\vec{e}_{1}$ & $\overrightarrow{0}$ & $-\vec{e}_{3}$ & $-\vec{e}_{2}$ \\
\hline$\vec{e}_{6}$ & $-\vec{e}_{7}$ & $\vec{e}_{4}$ & $-\vec{e}_{5}$ & $-\vec{e}_{2}$ & $\vec{e}_{3}$ & $\overrightarrow{0}$ & $\vec{e}_{1}$ \\
\hline$\vec{e}_{7}$ & $\vec{e}_{6}$ & $-\vec{e}_{5}$ & $-\vec{e}_{4}$ & $\vec{e}_{3}$ & $\vec{e}_{2}$ & $-\vec{e}_{1}$ & $\overrightarrow{0}$ \\
\hline
\end{tabular}

TABLE 2. Vector cross product multiplication table in $\mathbb{R}^{7}$.

From this table, by bilinearity, we obtain the cross product of any two vectors $\vec{p}, \vec{q} \in \mathbb{R}^{7}$,

$$
\vec{p} \times \vec{q}=\left(\sum_{i=1}^{7} p_{i} \overrightarrow{e_{i}}\right) \times\left(\sum_{i=1}^{7} q_{i} \overrightarrow{e_{i}}\right) .
$$

b) The matrix procedure in [8] is as follows.

$$
\vec{p} \times \vec{q}=M_{\vec{p}} \vec{q}=\left[\begin{array}{rrrrrrr}
0 & -p_{3} & p_{2} & -p_{5} & p_{4} & -p_{7} & p_{6} \\
p_{3} & 0 & -p_{1} & -p_{6} & p_{7} & p_{4} & -p_{5} \\
-p_{2} & p_{1} & 0 & p_{7} & p_{6} & -p_{5} & -p_{4} \\
p_{5} & p_{6} & -p_{7} & 0 & -p_{1} & -p_{2} & p_{3} \\
-p_{4} & -p_{7} & -p_{6} & p_{1} & 0 & p_{3} & p_{2} \\
p_{7} & -p_{4} & p_{5} & p_{2} & -p_{3} & 0 & -p_{1} \\
-p_{6} & p_{5} & p_{4} & -p_{3} & -p_{2} & p_{1} & 0
\end{array}\right]\left[\begin{array}{l}
q_{1} \\
q_{2} \\
q_{3} \\
q_{4} \\
q_{5} \\
q_{6} \\
q_{7}
\end{array}\right]
$$

iii) Triple scalar product

$$
\text { As } \vec{p} \times \vec{q} \bullet \vec{r}=\vec{r} \bullet \vec{p} \times \vec{q} \text {, we write }
$$

$$
\vec{p} \times \vec{q} \bullet \vec{r}=\left[\begin{array}{c}
r_{1} \\
r_{2} \\
r_{3} \\
r_{4} \\
r_{5} \\
r_{6} \\
r_{7}
\end{array}\right]^{T}\left[\begin{array}{rrrrrrr}
0 & -p_{3} & p_{2} & -p_{5} & p_{4} & -p_{7} & p_{6} \\
p_{3} & 0 & -p_{1} & -p_{6} & p_{7} & p_{4} & -p_{5} \\
-p_{2} & p_{1} & 0 & p_{7} & p_{6} & -p_{5} & -p_{4} \\
p_{5} & p_{6} & -p_{7} & 0 & -p_{1} & -p_{2} & p_{3} \\
-p_{4} & -p_{7} & -p_{6} & p_{1} & 0 & p_{3} & p_{2} \\
p_{7} & -p_{4} & p_{5} & p_{2} & -p_{3} & 0 & -p_{1} \\
-p_{6} & p_{5} & p_{4} & -p_{3} & -p_{2} & p_{1} & 0
\end{array}\right]\left[\begin{array}{l}
q_{1} \\
q_{2} \\
q_{3} \\
q_{4} \\
q_{5} \\
q_{6} \\
q_{7}
\end{array}\right] .
$$


In what follows, we use the standard notation $G(\vec{p}, \vec{q})$ for the Gram determinant of $\vec{p}$ and $\vec{q}$.

Proposition 2.4. [15] For the vectors $\vec{p}, \vec{q}, \vec{r} \in \mathbb{R}^{7}$, the following holds:

1. $\vec{p} \times \vec{q}=\overrightarrow{0}$ if and only if $\vec{p}$ and $\vec{q}$ are linearly dependent;

2. $\vec{p} \times \vec{q} \bullet \vec{r}=0$, whenever $\vec{p}, \vec{q}, \vec{r}$ are linearly dependent;

3. $(\vec{p} \times \vec{q}) \bullet(\vec{p} \times \vec{q})=\left|\begin{array}{cc}\vec{p} \bullet \vec{p} & \vec{p} \bullet \vec{q} \\ \vec{q} \bullet \vec{p} & \vec{q} \bullet \vec{q}\end{array}\right|=G(\vec{p}, \vec{q})$.

Proposition 2.5. [8] For any two octonions $p=p_{0}+\vec{p}$ and $q=q_{0}+\vec{q}$,

$$
p * q=p_{0} q_{0}-\vec{p} \bullet \vec{q}+q_{0} \vec{p}+p_{0} \vec{q}+\vec{p} \times \vec{q} .
$$

From this proposition and recalling some of the properties listed in the end of Subsection 2.2 we obtain the following identities.

Corollary 2.6. We have

$$
\begin{array}{ll}
\vec{p} * \vec{q}=-\vec{p} \bullet \vec{q}+\vec{p} \times \vec{q}, & \vec{p} * \vec{p}=-\vec{p} \bullet \vec{p}=-\|\vec{p}\|^{2}=-N(\vec{p}), \\
p * p^{-1}=1(p \neq 0), & \vec{p} *(\vec{p})^{-1}=1(\vec{p} \neq \overrightarrow{0}), \\
\gamma * p=\gamma p_{0}+\gamma \vec{p}, \text { with } \gamma \in \mathbb{R} \quad \text { and } & \gamma * \vec{p}=\gamma \vec{p}, \text { with } \gamma \in \mathbb{R} .
\end{array}
$$

The subsequent result justifies the equivalence between the two first representations for a straight line over the octonions in Section 3.

Corollary 2.7. For any pure octonions $\vec{p}$ and $\vec{q}, \vec{p}$ and $\vec{q}$ have parallel vector parts if and only if $\vec{p}$ and $\vec{q}$ are parallel.

Proof. If $\vec{V}(\vec{p})=\alpha \vec{V}(\vec{q})$ for some $\alpha \in \mathbb{R}$ then $\vec{V}\left(\vec{p} *(\vec{q})^{c}\right)=\vec{V}\left(\alpha \vec{q} *(\vec{q})^{c}\right)=$ $\vec{V}(\alpha N(\vec{q}))=\overrightarrow{0}$. Hence, $\vec{p}$ and $\vec{q}$ are parallel octonions.

Suppose now that $\vec{V}\left(\vec{p} *(\vec{q})^{c}\right)=\overrightarrow{0}$. So, by Proposition $2.5, \vec{p} \times(\vec{q})^{c}=\overrightarrow{0}$. Then $\vec{p} \times \vec{q}=\overrightarrow{0}$. Thus, $\vec{p}=\beta \vec{q}$ for some $\beta \in \mathbb{R}$.

\subsection{Double vector cross product in $\mathbb{R}^{7}$}

The vector cross product in $\mathbb{R}^{7}$ is not associative, so we need to take a substitute, in some sense, for the associative law. This can be done, considering the double vector cross product in $\mathbb{R}^{7}$, through an extension of the Grassmann identity in $\mathbb{R}^{3},[5]$.

Theorem 2.8. [9] For $\vec{p}, \vec{q}, \vec{r} \in \mathbb{R}^{7}$, we have

$$
\vec{p} \times(\vec{q} \times \vec{r})=\vec{q}(\vec{p} \bullet \vec{r})-\vec{r}(\vec{p} \bullet \vec{q})+\frac{1}{3} J(\vec{p}, \vec{q}, \vec{r}),
$$

where the Jacobian $J$ is the alternate trilinear application defined by

$$
J(\vec{p}, \vec{q}, \vec{r})=\vec{p} \times(\vec{q} \times \vec{r})+\vec{q} \times(\vec{r} \times \vec{p})+\vec{r} \times(\vec{p} \times \vec{q}) .
$$

Corollary 2.9. For vectors in $\mathbb{R}^{7}$ the following holds:

1. $\vec{p} \times(\vec{q} \times \vec{p})=\vec{q}(\vec{p} \bullet \vec{p})-\vec{p}(\vec{p} \bullet \vec{q})$;

2. $\left[(\vec{p} \times \vec{q}) *(\vec{q})^{-1}\right] \times \vec{q}=\vec{p} \times \vec{q} \quad(\vec{q} \neq \overrightarrow{0})$. 
Proof. The result (1) is an immediate consequence of the previous theorem. As far as (2), invoking the alternativity of octonions, we have

$$
\begin{aligned}
\left(\vec{a} *(\vec{q})^{-1}\right) \times \vec{q} & =\vec{V}\left(\left(\vec{a} *(\vec{q})^{-1}\right) * \vec{q}\right) \\
& =-\frac{1}{N(\vec{q})} \vec{V}((\vec{a} * \vec{q}) * \vec{q}) \\
& =-\frac{1}{N(\vec{q})} \vec{V}(\vec{a} *(\vec{q} * \vec{q})) \\
& =\vec{V}\left(\vec{a} *\left((q)^{-1} * \vec{q}\right)\right) \\
& =\vec{V}(\vec{a}) .
\end{aligned}
$$

The proof ends taking $\vec{a}=\vec{p} \times \vec{q}$, since $\vec{V}(\vec{a})=\vec{a}$.

\section{Point of Minimum Euclidean Norm of the Octonion Line}

An extension to the octonionic context is made of what appears in [16] concerning the quaternion equation of a straight line. However, the nonassociativity of the octonions has to be taken into account.

We write the octonion equation of a line and then look for the point onto this line which is at the shortest distance from $\mathcal{O}$, the origin of the coordinates.

In what follows, taking into account the identification of $\mathbb{R}^{7}$ with $\operatorname{Im} \mathbb{D}$, we consider the vectors $\vec{a}, \vec{b}, \vec{x} \in \mathbb{R}^{7}, \vec{b} \neq \overrightarrow{0}$, as pure octonions, i.e., $\vec{a}, \vec{b}, \vec{x}$ are the vector parts of the pure octonions $a, b, x$. Moreover, in the present section and in the following ones, through a vectorization of the affine space $\mathbb{R}^{7}$, the end-point of a position vector (with respect to $\mathcal{O}$ ) in $\mathbb{R}^{7}$ is identified with that vector.

An equation of the line passing through the end-point of the position vector $\vec{a}$ with direction $\vec{b}$ is

$$
\vec{x}=\vec{a}+\alpha \vec{b}, \quad \alpha \in \mathbb{R},
$$

where $\vec{x}$ is the position vector of the generic point on the line with respect to the origin $\mathcal{O}$. The pure octonions $\vec{x}-\vec{a}$ and $\vec{b}$ have parallel vector parts if and only if $\vec{V}\left[(\vec{x}-\vec{a}) *(\vec{b})^{c}\right]=\overrightarrow{0}$, this being equivalent to $(\vec{x}-\vec{a}) \times \vec{b}=\overrightarrow{0}$. Hence

$$
\vec{x} \times \vec{b}=\vec{a} \times \vec{b}
$$

is another representation of the line given in (3.1).

Concerning the problem of finding the point of the straight line (3.1) having minimum Euclidean norm, by using octonions, we must state that we are mainly interested in the construction method, since the existence and uniqueness of this point is guaranteed by the following result.

Theorem 3.1. [10, Thm. 1, page 64] Let $T$ be a subspace of $\mathbb{R}^{n}, \vec{a}$ a fixed element in $\mathbb{R}^{n}$ and consider the linear variety $\vec{a}+T$ denoted by $V$. Then there is a unique vector $\vec{s}$ in $V$ of minimum Euclidean norm. Furthermore, $\vec{s}$ is orthogonal to $T$.

In order to find the above mentioned point, the representation (3.2) is more adequate. 
Proposition 3.2. Let us consider the octonion equation (3.2) of a straight line. Then

1. another representation of the line in $(3.2)$ is $\vec{x}=(-\vec{b} \bullet \vec{x}+\vec{a} \times \vec{b}) *(\vec{b})^{-1}$;

2. $N(\vec{x})=\frac{(-\vec{b} \bullet \vec{x})^{2}}{N(\vec{b})}+N\left[(\vec{a} \times \vec{b}) *(\vec{b})^{-1}\right]$;

3. the octonions $(\vec{a} \times \vec{b}) *(\vec{b})^{-1}$ and $\vec{b}$ are orthogonal.

Proof. (1) Multiplying both members of equation (3.2), on the right, by the pure octonion $(\vec{b})^{-1}$, we arrive at $(\vec{x} \times \vec{b}) *(\vec{b})^{-1}=\vec{M} *(\vec{b})^{-1}$, where, here and in the rest of the proof, $\vec{M}=\vec{a} \times \vec{b}$ is a pure octonion. We have

$$
\begin{aligned}
(\vec{x} \times \vec{b}) *(\vec{b})^{-1} & =-(\vec{x} \times \vec{b}) \bullet(\vec{b})^{-1}+(\vec{x} \times \vec{b}) \times(\vec{b})^{-1} \\
& =-\frac{1}{N(\vec{b})}[-(\vec{x} \times \vec{b}) \bullet \vec{b}+(\vec{x} \times \vec{b}) \times \vec{b}] \\
& =-\frac{1}{N(\vec{b})}[0-\vec{b} \times(\vec{x} \times \vec{b})] \\
& =-\frac{1}{N(\vec{b})}[-\vec{x}(\vec{b} \bullet \vec{b})+\vec{b}(\vec{b} \bullet \vec{x})] \\
& =-\frac{1}{N(\vec{b})}[-N(\vec{b}) \vec{x}+(\vec{b} \bullet \vec{x}) \vec{b}] \\
& =\vec{x}-\frac{(\vec{b} \bullet \vec{x})}{N(\vec{b})} \vec{b} \\
& =\vec{x}+(\vec{b} \bullet \vec{x})(\vec{b})^{-1}
\end{aligned}
$$

Thus, we conclude that $\vec{x}=(-\vec{b} \bullet \vec{x}+\vec{M}) *(\vec{b})^{-1}$.

(2) Let us put $\gamma=-\vec{b} \bullet \vec{x}$. We get

$$
\begin{aligned}
N(\vec{x}) & =\left[\gamma *(\vec{b})^{-1}+\vec{M} *(\vec{b})^{-1}\right] *\left[\gamma *(\vec{b})^{-1}+\vec{M} *(\vec{b})^{-1}\right]^{c} \\
& =\left[\gamma(\vec{b})^{-1}+\vec{M} *(\vec{b})^{-1}\right] *\left[\gamma(\vec{b})^{-1}+\vec{M} *(\vec{b})^{-1}\right]^{c} \\
& =\left[\gamma(\vec{b})^{-1}+\vec{M} *(\vec{b})^{-1}\right] *\left[(-\gamma)(\vec{b})^{-1}+(\vec{b})^{-1} * \vec{M}\right] \\
& =\left(-\gamma^{2}\right)(\vec{b})^{-1} *(\vec{b})^{-1}+\left[\vec{M} *(\vec{b})^{-1}\right] *\left[(\vec{b})^{-1} * \vec{M}\right] \\
& =\frac{\gamma^{2}}{N(\vec{b})}+N\left[\vec{M} *(\vec{b})^{-1}\right] .
\end{aligned}
$$

(3) By the alternativity of octonions, we have

$$
\left[\vec{M} *(\vec{b})^{-1}\right] *(\vec{b})^{c}=\frac{1}{N(\vec{b})}[\vec{M} * \vec{b}] * \vec{b}=\frac{1}{N(\vec{b})} \vec{M} *[\vec{b} * \vec{b}]=-\vec{M} *\left[(\vec{b})^{-1} * \vec{b}\right] .
$$

Whence, $\left[\vec{M} *(\vec{b})^{-1}\right] *(\vec{b})^{c}=-\vec{M}$ with $S(-\vec{M})=0$.

We are now in conditions to give an answer to the problem stated in the beginning of this section.

Proposition 3.3. Let $l$ be the line given by

$$
\vec{x}=\vec{a}+\alpha \vec{b}, \alpha \in \mathbb{R},
$$

or, equivalently, by

$$
\vec{x} \times \vec{b}=\vec{a} \times \vec{b},
$$

where $\vec{a}, \vec{b}, \vec{x} \in \operatorname{Im}\left(\mathbb{D}\right.$ with $\vec{b} \neq \overrightarrow{0}$. Then $\vec{s}=(\vec{a} \times \vec{b}) *(\vec{b})^{-1}$ is the point on the line which is nearest to the origin of the coordinates. 
Proof. We look for a pure octonion $\vec{s}$ orthogonal to the pure octonion $\vec{b}$. From assertion (2) in Proposition 3.2, we get the minimum norm octonion $\vec{x}$ if and only if $\vec{x}$ is orthogonal to $\vec{b}$, that is, $S\left(\vec{x} *(\vec{b})^{c}\right)=\vec{x} \bullet \vec{b}=0$. But assertion (3) in the same proposition states that $(\vec{a} \times \vec{b}) *(\vec{b})^{-1}$ and $\vec{b}$ are orthogonal. Thus, it must be

$$
\vec{s}= \pm(\vec{a} \times \vec{b}) *(\vec{b})^{-1} .
$$

But $-(\vec{a} \times \vec{b}) *(\vec{b})^{-1}$ does not belong to the line in (3.3). Indeed, we have

$$
\begin{aligned}
-(\vec{a} \times \vec{b}) *(\vec{b})^{-1} & =-\left[-(\vec{a} \times \vec{b}) \bullet(\vec{b})^{-1}+(\vec{a} \times \vec{b}) \times(\vec{b})^{-1}\right] \\
& =\frac{1}{N(\vec{b})}[-(\vec{a} \times \vec{b}) \bullet \vec{b}+(\vec{a} \times \vec{b}) \times \vec{b}] \\
& =\frac{1}{N(\vec{b})}(\vec{a} \times \vec{b}) \times \vec{b} .
\end{aligned}
$$

Henceforth,

$$
\begin{aligned}
-\left[(\vec{a} \times \vec{b}) *(\vec{b})^{-1}\right] \times \vec{b} & =\frac{1}{N(\vec{b})}((\vec{a} \times \vec{b}) \times \vec{b}) \times \vec{b} \\
& =\left(-\frac{1}{N(\vec{b})}\right) \vec{b} \times((\vec{a} \times \vec{b}) \times \vec{b}) \\
& =-\frac{1}{N(\vec{b})}[(\vec{a} \times \vec{b})(\vec{b} \bullet \vec{b})-\vec{b}(\vec{b} \bullet(\vec{a} \times \vec{b}))] \\
& =-\frac{1}{N(\vec{b})} N(\vec{b})(\vec{a} \times \vec{b}) \\
& =-\vec{a} \times \vec{b}
\end{aligned}
$$

We conclude that the pure octonion $(\vec{a} \times \vec{b}) *(\vec{b})^{-1}$ is the orthogonal projection of the origin onto the line $l$.

Corollary 3.4. The Euclidean distance $d(\mathcal{O}, l)$ between the origin of the coordinates and the line $l$ defined by

$$
\vec{x}=\vec{a}+\alpha \vec{b}, \alpha \in \mathbb{R},
$$

where $\vec{a}, \vec{b}, \vec{x} \in \operatorname{Im} \mathbb{D}$ with $\vec{b} \neq \overrightarrow{0}$, is given by

$$
d^{2}(\mathcal{O}, l)=\frac{G(\vec{a}, \vec{b})}{N(\vec{b})} .
$$

Proof. Invoking the Moufang identity (2.1), we obtain

$$
\begin{aligned}
N\left[(\vec{a} \times \vec{b}) *(\vec{b})^{-1}\right] & =\left[(\vec{a} \times \vec{b}) *(\vec{b})^{-1}\right] *\left[(\vec{a} \times \vec{b}) *(\vec{b})^{-1}\right]^{c} \\
& =\left[(\vec{a} \times \vec{b}) *(\vec{b})^{-1}\right] *\left[(\vec{b})^{-1} *(\vec{a} \times \vec{b})\right] \\
& =(\vec{a} \times \vec{b}) *\left[(\vec{b})^{-1} *(\vec{b})^{-1}\right] *(\vec{a} \times \vec{b}) \\
& =\left(-\frac{1}{N(\vec{b})}\right)(\vec{a} \times \vec{b}) *(\vec{a} \times \vec{b}) \\
& =-\frac{1}{N(\vec{b})}[-(\vec{a} \times \vec{b}) \bullet(\vec{a} \times \vec{b})+(\vec{a} \times \vec{b}) \times(\vec{a} \times \vec{b})] \\
& =\frac{1}{N(\vec{b})}(\vec{a} \times \vec{b}) \bullet(\vec{a} \times \vec{b}) \\
& =\frac{G(\vec{a}, \vec{b})}{N(\vec{b})} .
\end{aligned}
$$




\section{Projection of an External Point onto a Line on the Octonions}

In Proposition 3.3, we obtained the projection of the origin of the coordinates onto an octonion line. In order to reach the orthogonal projection of any point onto a given straight line, we will make no use of translations. To go on with this alternative procedure, we will need the following preliminary result (see [7, Théo. 2.2.5, page 45] or [10, Theo. 2, page 51] for a more general result).

Theorem 4.1. Let $T$ be a subspace of $\mathbb{R}^{n}, \vec{p}$ a fixed element in $\mathbb{R}^{n}$ and consider the linear variety $\vec{p}+T$ denoted by $V$. A necessary and sufficient condition that $\vec{q} \in V$ be the best approximation of $\vec{m} \notin V$ onto $V$ is that $\vec{q}-\vec{m}$ be orthogonal to $T(\vec{q}-\vec{m} \perp T)$.

Proposition 4.2. Let us consider the line

$$
l_{\vec{p}}: \quad \vec{x}=\vec{p}+\alpha \vec{u}, \quad \vec{p}, \vec{x}, \vec{u} \in \operatorname{Im} \mathbb{D} \text { with } \vec{u} \neq \overrightarrow{0}, \alpha \in \mathbb{R},
$$

a given point $\vec{m}$ external to $l_{\vec{p}}$ and the line

$$
l_{\vec{m}}: \quad \vec{x}=\vec{m}+\beta \vec{u}, \quad \vec{m}, \vec{x}, \vec{u} \in \operatorname{Im} \mathbb{D} \text { with } \vec{u} \neq \overrightarrow{0}, \beta \in \mathbb{R} .
$$

Then the orthogonal projection $\mathbb{P}_{l_{\vec{p}}}(\vec{m})$ of the point $\vec{m}$ onto $l_{\vec{p}}$ is given by

$$
\vec{s}_{\vec{p}}=\vec{x}_{\vec{p}}-\vec{x}_{\vec{m}}+\vec{m},
$$

where $\vec{x}_{\vec{p}}$ and $\vec{x}_{\vec{m}}$ are, respectively, the orthogonal projections of $\mathcal{O}$, the origin of the coordinates, onto the lines $l_{\vec{p}}$ and $l_{\vec{m}}$.

Proof. Using Proposition 3.3, we first compute $\vec{x}_{\vec{p}}$ and $\vec{x}_{\vec{m}}$. We have

$$
\vec{x}_{\vec{p}}=(\vec{p} \times \vec{u}) *(\vec{u})^{-1} \quad \text { and } \quad \vec{x}_{\vec{m}}=(\vec{m} \times \vec{u}) *(\vec{u})^{-1} .
$$

By Proposition 3.2,

$$
\vec{x}_{\vec{p}}, \vec{x}_{\vec{m}} \perp \vec{u} .
$$

Let us take $\vec{w}=\vec{m}-\vec{x}_{\vec{m}}$ and consider

$$
\vec{s}_{\vec{p}}=\vec{x}_{\vec{p}}+\vec{w}=\vec{x}_{\vec{p}}+\vec{m}-\vec{x}_{\vec{m}} .
$$

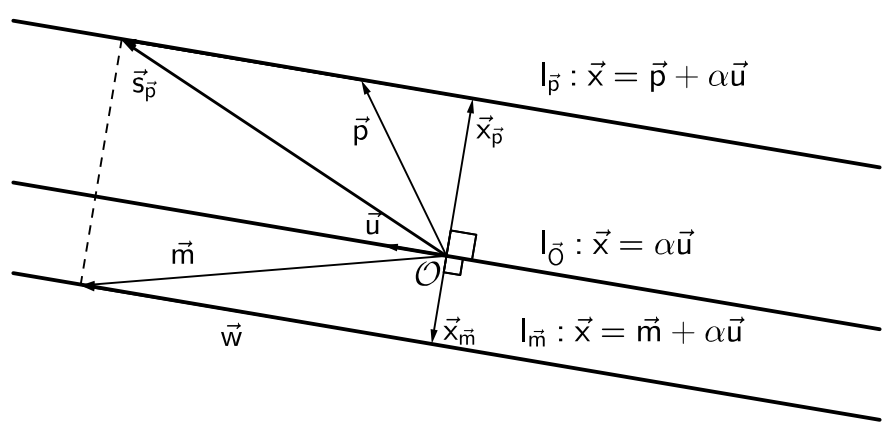

FiguRE 2. $\vec{s}_{\vec{p}}$, the orthogonal projection of $\vec{m}$ onto $l_{\vec{p}}$. 
According to the Theorem 4.1, in order to prove that $\vec{s}_{\vec{p}}=\mathbb{P}_{l_{\vec{p}}}(\vec{m})$, we must show that

$$
\vec{s}_{\vec{p}}-\vec{m} \perp \vec{u}
$$

We have:

$$
\left(\vec{s}_{\vec{p}}-\vec{m}\right) *(\vec{u})^{c}=\left(\vec{x}_{\vec{p}}-\vec{x}_{\vec{m}}\right) *(\vec{u})^{c}=\vec{x}_{\vec{p}} *(\vec{u})^{c}-\vec{x}_{\vec{m}} *(\vec{u})^{c} .
$$

Due to (4.1), it is possible to write

$$
S\left(\vec{x}_{\vec{p}} *(\vec{u})^{c}\right)=0=S\left(\vec{x}_{\vec{m}} *(\vec{u})^{c}\right) .
$$

Thus, $S\left[\left(\vec{s}_{\vec{p}}-\vec{m}\right) *(\vec{u})^{c}\right]=0$.

Remark 4.3. $\vec{s}_{\vec{p}}$ is, in fact, the projection of the point $\vec{m}$ onto the line $l_{\vec{p}}$. On the one hand, Theorem 3.1 assures us that $\vec{s}_{\vec{p}}$ is orthogonal to $\vec{u}$. On the other hand, recalling the result (2) of Corollary 2.9, we have

$$
\begin{aligned}
\vec{s}_{\vec{p}} \times \vec{u} & =\left(\vec{x}_{\vec{p}}+\vec{m}-\vec{x}_{\vec{m}}\right) \times \vec{u}=\overrightarrow{x_{P}} \times \vec{u}-\vec{x}_{\vec{m}} \times \vec{u}+\vec{m} \times \vec{u} . \\
& =\left[(\vec{p} \times \vec{u}) *(\vec{u})^{-1}\right] \times \vec{u}-\left[(\vec{m} \times \vec{u}) *(\vec{u})^{-1}\right] \times \vec{u}+\vec{m} \times \vec{u} \\
& =\vec{p} \times \vec{u}-\vec{m} \times \vec{u}+\vec{m} \times \vec{u}=\vec{p} \times \vec{u},
\end{aligned}
$$

proving that $\vec{s}_{\vec{p}} \in l_{\vec{p}}$, according to the alternative equation $\vec{x} \times \vec{u}=\vec{p} \times \vec{u}$ of $l_{\vec{p}}$.

Example. Let us consider the line

$$
l_{\vec{p}}: \quad \vec{x}=\vec{p}+\alpha \vec{u}, \alpha \in \mathbb{R},
$$

where $\vec{p}=\left[\begin{array}{lllllll}1 & 1 & 1 & 1 & 1 & 1 & 1\end{array}\right]^{T}$ and $\vec{u}=\left[\begin{array}{lllllll}2 & 3 & 4 & 1 & 3 & -1 & 2\end{array}\right]^{T}$, the point

$$
\vec{m}=\left[\begin{array}{lllllll}
0 & 1 & 0 & 1 & 0 & 1 & 0
\end{array}\right]^{T}
$$

external to $l_{\vec{p}}$ and the line $l_{\vec{m}}: \quad \vec{x}=\vec{m}+\beta \vec{u}, \beta \in \mathbb{R}$. Using Proposition 4.2,

$$
\mathbb{P}_{l_{\vec{p}}}(\vec{m})=\vec{s}_{\vec{p}}=\vec{x}_{\vec{p}}-\vec{x}_{\vec{m}}+\vec{m}=-\frac{1}{N(\vec{u})}[(\vec{p}-\vec{m}) \times \vec{u}] * \vec{u}+\vec{m} .
$$

As $(\vec{p}-\vec{m}) \times \vec{u}$ is given by

$$
M_{\vec{p}-\vec{m}} \vec{u}=\left[\begin{array}{rrrrrrr}
0 & -1 & 0 & -1 & 0 & -1 & 0 \\
1 & 0 & -1 & 0 & 1 & 0 & -1 \\
0 & 1 & 0 & 1 & 0 & -1 & 0 \\
1 & 0 & -1 & 0 & -1 & 0 & 1 \\
0 & -1 & 0 & 1 & 0 & 1 & 0 \\
1 & 0 & 1 & 0 & -1 & 0 & -1 \\
0 & 1 & 0 & -1 & 0 & 1 & 0
\end{array}\right]\left[\begin{array}{r}
2 \\
3 \\
4 \\
1 \\
3 \\
-1 \\
2
\end{array}\right]=\left[\begin{array}{r}
-3 \\
-1 \\
5 \\
-3 \\
-3 \\
1 \\
1
\end{array}\right],
$$

then 


$$
\begin{aligned}
& \mathbb{P}_{l_{\vec{p}}}(\vec{m})=-\frac{1}{44}\left[\begin{array}{rrrrrrrr}
0 & 3 & 1 & -5 & 3 & 3 & -1 & -1 \\
-3 & 0 & -5 & -1 & 3 & -3 & -1 & 1 \\
-1 & 5 & 0 & 3 & -1 & 1 & -3 & 3 \\
5 & 1 & -3 & 0 & 1 & 1 & 3 & 3 \\
-3 & -3 & 1 & -1 & 0 & 3 & 1 & 5 \\
-3 & 3 & -1 & -1 & -3 & 0 & 5 & -1 \\
1 & 1 & 3 & -3 & -1 & -5 & 0 & 3 \\
1 & -1 & -3 & -3 & -5 & 1 & -3 & 0
\end{array}\right]\left[\begin{array}{r}
0 \\
2 \\
3 \\
4 \\
1 \\
3 \\
-1 \\
2
\end{array}\right]+\left[\begin{array}{l}
0 \\
0 \\
1 \\
0 \\
1 \\
0 \\
1 \\
0
\end{array}\right] \\
&= {\left[\begin{array}{lrllllll}
0 & \frac{1}{2} & \frac{1}{4} & 0 & \frac{3}{4} & \frac{1}{4} & \frac{5}{4} & \frac{1}{2}
\end{array}\right]^{T} . }
\end{aligned}
$$

\section{Distance between Two Skew Lines over the Octonions}

The distance between two given (skew) lines $l_{\vec{p}}$ and $l_{\vec{q}}$ is the length of the shortest segment connecting the two lines. In order to find it, in this section, the main role is played by the generic point of each line.

Proposition 5.1. Let be given two skew lines over the octonions,

$$
l_{\vec{p}}: \quad \vec{x}=\vec{p}+\alpha \vec{u} \quad \text { and } \quad l_{\vec{q}}: \quad \vec{x}=\vec{q}+\beta \vec{v},
$$

where $\vec{p}, \vec{q}, \vec{x}, \vec{u}, \vec{v}$ are pure octonions with $\vec{u} \neq \overrightarrow{0}$ and $\vec{v} \neq \overrightarrow{0}, \alpha, \beta \in \mathbb{R}$. Then, the best approximation pair

$$
\left(\vec{s}_{\vec{p}}, \vec{s}_{\vec{q}}\right) \in l_{\vec{p}} \times l_{\vec{q}}
$$

of points which are the extremities of the shortest line segment connecting the lines $l_{\vec{p}}$ and $l_{\vec{q}}$ is given by

$$
\left(\vec{s}_{\vec{p}}\left(\beta^{*}\right), \vec{s}_{\vec{q}}\left(\alpha^{*}\right)\right) \text {, that is, }\left(\vec{p}+\alpha^{*} \vec{u}, \vec{q}+\beta^{*} \vec{v}\right),
$$

where $\left(\alpha^{*}, \beta^{*}\right) \in \mathbb{R} \times \mathbb{R}$ is the unique exact solution of the overdetermined consistent system

$$
\left\{\begin{array}{l}
\vec{s}_{\vec{p}}(\beta)=\vec{p}_{G}(\alpha) \\
\vec{s}_{\vec{q}}(\alpha)=\vec{q}_{G}(\beta)
\end{array},\right.
$$

with $\vec{p}_{G}(\alpha)$ and $\vec{q}_{G}(\beta)$ generic elements of the lines $l_{\vec{p}}$ and $l_{\vec{q}}$, respectively.

Proof. This can basically be described as a doubling procedure of projection of two generic points, each one belonging to the respective line, and such that each one is projected on the other following the method pointed in Section 4. Thus, in order to solve (5.1), we must find generic elements $\vec{p}_{G}(\alpha) \in l_{\vec{p}}$ and $\vec{q}_{G}(\beta) \in l_{\vec{q}}$ such that $\vec{p}_{G}(\alpha)$ (resp., $\vec{q}_{G}(\beta)$ ) is the orthogonal projection of $\vec{q}_{G}(\beta)$ (resp., $\left.\vec{p}_{G}(\alpha)\right)$ on $l_{\vec{p}}$ (resp., on $l_{\vec{q}}$ ).

Corollary 5.2. The best approximation points of the lines $l_{\vec{p}}$ and $l_{\vec{q}}$ are $\vec{p}+$ $\alpha^{*} \vec{u} \in l_{\vec{p}}$ and $\vec{q}+\beta^{*} \vec{v} \in l_{\vec{q}}$, where the pair $\left(\alpha^{*}, \beta^{*}\right) \in \mathbb{R} \times \mathbb{R}$ is given by

$$
\left\{\begin{array}{l}
(\vec{p} \times \vec{u}) *(\vec{u})^{-1}-\left(\vec{q}_{G}(\beta) \times \vec{u}\right) *(\vec{u})^{-1}+\vec{q}_{G}(\beta)=\vec{p}_{G}(\alpha) \\
(\vec{q} \times \vec{v}) *(\vec{v})^{-1}-\left(\vec{p}_{G}(\alpha) \times \vec{u}\right) *(\vec{v})^{-1}+\vec{p}_{G}(\alpha)=\vec{q}_{G}(\beta) .
\end{array}\right.
$$

Proof. Applying Proposition 4.2, it is possible to observe that the simultaneous equations (5.1) are equivalent to (5.2). 
Corollary 5.3. The best approximation points of the lines $l_{\vec{p}}$ and $l_{\vec{q}}$ are $\vec{p}+$ $\alpha^{*} \vec{u} \in l_{\vec{p}}$ and $\vec{q}+\beta^{*} \vec{v} \in l_{\vec{q}}$, where the pair $\left(\alpha^{*}, \beta^{*}\right) \in \mathbb{R} \times \mathbb{R}$ is obtained from

$$
\left\{\begin{array}{l}
\vec{p}-\frac{\vec{p} \bullet \vec{u}}{N(\vec{u})} \vec{u}+\frac{\vec{q}_{G}(\beta) \bullet \vec{u}}{N(\vec{u})} \vec{u}=\vec{p}_{G}(\alpha) \\
\vec{q}-\frac{\vec{q} \bullet \vec{v}}{N(\vec{v})} \vec{v}+\frac{\vec{p}_{G}(\alpha) \bullet \vec{v}}{N(\vec{v})} \vec{v}=\vec{q}_{G}(\beta) .
\end{array}\right.
$$

Proof. Analogously to a part of the proof of (1) in Proposition 3.2, we deduce

$$
(\vec{a} \times \vec{b}) *(\vec{b})^{-1}=\vec{a}-\frac{\vec{a} \bullet \vec{b}}{N(\vec{b})} \vec{b} .
$$

Thus, the system (5.2) can be written as (5.3).

Remark 5.4. The consistency of the system is a consequence of a so-called Classical Projection Theorem [10, page 51] on Hilbert spaces. It can also result as a double and simultaneous application of Theorem 4.1.

Example. Let us consider two skew lines

$$
l_{\vec{p}}: \quad \vec{x}=\vec{p}+\alpha \vec{u}, \alpha \in \mathbb{R} \quad \text { and } \quad l_{\vec{q}}: \quad \vec{x}=\vec{q}+\beta \vec{v}, \beta \in \mathbb{R},
$$

where

$$
\vec{p}=\left[\begin{array}{lllllll}
1 & 1 & 1 & 1 & 1 & 1 & 1
\end{array}\right]^{T}, \vec{u}=\left[\begin{array}{lllllll}
2 & 3 & 4 & 1 & 3 & -1 & 2
\end{array}\right]^{T},
$$

and

$$
\vec{q}=\left[\begin{array}{lllllll}
0 & 1 & 0 & 1 & 0 & 1 & 0
\end{array}\right]^{T}, \vec{v}=\left[\begin{array}{lllllll}
0 & 1 & 0 & -1 & 0 & 1 & 0
\end{array}\right]^{T} .
$$

In order to apply Corollary 5.3 , we have $N(\vec{u})=44, N(\vec{v})=3, \vec{p} \bullet \vec{u}=14$ and $\vec{q} \bullet \vec{v}=1$. Further, since

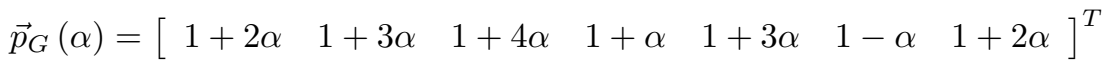

and

$$
\vec{q}_{G}(\beta)=\left[\begin{array}{lllllll}
0 & 1+\beta & 0 & 1-\beta & 0 & 1+\beta & 0
\end{array}\right]^{T},
$$

we have $\vec{q}_{G}(\beta) \bullet \vec{u}=3+\beta$ and $\vec{p}_{G}(\alpha) \bullet \vec{v}=1+\alpha$. Replacing in (5.3) and solving the system, we get:

$$
\alpha^{*}=-\frac{33}{131} \quad \text { and } \quad \beta^{*}=-\frac{11}{131} .
$$

Finally, the best approximation pair is $\left(\vec{s}_{\vec{p}}, \vec{s}_{\vec{q}}\right)$, where

$$
\vec{s}_{\vec{p}}=\vec{p}+\left(-\frac{33}{131}\right) \vec{u}=\left[\begin{array}{lllllll}
\frac{65}{131} & \frac{32}{131} & -\frac{1}{131} & \frac{98}{131} & \frac{32}{131} & \frac{164}{131} & \frac{65}{131}
\end{array}\right]^{T}
$$

and

$$
\vec{s}_{\vec{q}}=\vec{q}+\left(-\frac{11}{131}\right) \vec{v}=\left[\begin{array}{lllllll}
0 & \frac{120}{131} & 0 & \frac{142}{131} & 0 & \frac{120}{131} & 0
\end{array}\right]^{T} .
$$




\section{Acknowledgment}

P. Saraiva was supported by CMUC - Centre for Mathematics, University of Coimbra.

P.D. Beites was supported by FCT - Portuguese Foundation for Science and Technology ("Fundação para a Ciência e a Tecnologia", Portugal), through CMA-UBI - Centre for Mathematics and Applications, University of Beira Interior, within the project PEst-OE/MAT/UI0212/2011.

The work of C. Costa was partially supported by the Portuguese Government through FCT, Project PEst-C/CED/UI0194/2013-2014.

J. Vitória gratefully thanks the generous logistic support from the Department of Mathematics of the University of Coimbra and also asserts that, in part, this work was supported by Portuguese funds through the CIDMA - Center for Research and Development in Mathematics and Applications, and FCT, within project with reference PEst-OE/MAT/UI4106/2014.

\section{References}

[1] T. Appelquist, A. Chodos and P. G. O. Freund, Modern Kaluza-Klein Theories. Addison-Wesley, California, Los Angeles, 1987.

[2] N. Arkani-Hamed, S. Dimopoulos and G. R. Dvali, The Hierarchy Problem and New Dimensions at a Millimeter. Physics Letters B 429 (1998), 263-272.

[3] J. C. Baez, The Octonions. Bulletin of the American Mathematical Society 39 (2002), 145-205.

[4] R. B. Brown and A. Gray, Vector Cross Products. Commentarii Mathematici Helvetici 42 (1967), 222-236.

[5] H.-D. Ebbinghaus, H. Hermes, F. Hirzebruch, M. Koecher, K. Mainzer, J. Neukirch, A. Prestel and R. Remmert, Numbers. Springer, New York, 1995.

[6] N. Jacobson, Composition Algebras and their Automorphisms. Rendiconti del Circolo Matematico di Palermo 7 (1958), 55-80.

[7] P.-J. Laurent, Approximation et Optimisation. Hermann, Paris, 1972.

[8] F. S. Leite, The Geometry of Hypercomplex Matrices. Linear and Multilinear Algebra 34 (1993), 123-132.

[9] F. S. Leite and P. Crouch, The Triple Cross Product in $\mathbb{R}^{7}$. Reports on Mathematical Physics 39 (1997), 407-424.

[10] D. G. Luenberger, Optimization by Vector Space Methods. John Wiley and Sons, New York, 1969.

[11] K. L. McDonald, Warping the Universal Extra Dimensions. Physical Review D 80 (2009), 24-38.

[12] A. D. Medina and E. Pontón, Warped Radion Dark Matter. Journal of High Energy Physics 9 (2011), 1-46.

[13] S. Okubo, Introduction to Octonion and other Non-Associative Algebras in Physics. Cambridge University Press, New York, 2005.

[14] L. Randall and R. Sundrum, Large mass hierarchy from a small extra dimension. Physical Review Letters 83 (1999), 3370-3373. 
[15] R. Shaw, Vector cross products in $n$ dimensions. International Journal of Mathematical Education in Science and Technology 18 (1987), 803-816.

[16] J. P. Ward, Quaternions and Cayley Numbers. Kluwer, Dordrecht, 1997.

\section{P. Saraiva}

Mathematics Group of the Faculty of Economics and CMUC

Centre for Mathematics

University of Coimbra

Coimbra, Portugal

e-mail: psaraiva@fe.uc.pt

P.D. Beites

Department of Mathematics and CMA-UBI

Centre for Mathematics and Applications

University of Beira Interior

Covilhã, Portugal

e-mail: pbeites@ubi.pt

J. Fernandes

CGUC - Geophysical Institute

Department of Mathematics and Astronomical Observatory

University of Coimbra

Coimbra, Portugal

e-mail: jmfernan@mat.uc.pt

C. Costa

Universidade de Trás-os-Montes e Alto Douro, UTAD and

CIDTFF - Centro de Investigação "Didática e Tecnologia na Formação de Formadores" - Laboratório de Didática de Ciências e Tecnologia (UTAD)

Quinta de Prados, 5001-801Vila Real, Portugal

e-mail: mcosta@utad.pt

José Vitória

Department of Mathematics

University of Coimbra

Coimbra, Portugal

and

CIDMA - Center for Research and Development in Mathematics and Applications University of Aveiro

Aveiro, Portugal

e-mail: jvitoria@mat.uc.pt

Received: July 21, 2014.

Accepted: November 20, 2014. 